\title{
Increased lanosterol turnover: a metabolic burden for daunorubicin-resistant leukemia cells
}

\author{
Claudia Stäubert ${ }^{1,2,5} \cdot$ Rosanna Krakowsky $^{5} \cdot$ Hasanuzzaman Bhuiyan $^{3} \cdot$ \\ Barbara Witek ${ }^{1} \cdot$ Anna Lindahl $^{4} \cdot$ Oliver Broom $^{1} \cdot$ Anders Nordström $^{1,2,4}$
}

Received: 30 November 2015/Accepted: 10 December 2015/Published online: 23 December 2015

(C) The Author(s) 2015. This article is published with open access at Springerlink.com

\begin{abstract}
The cholesterol metabolism is essential for cancer cell proliferation. We found the expression of genes involved in the cholesterol biosynthesis pathway up-regulated in the daunorubicin-resistant leukemia cell line CEM/ $\mathrm{R} 2$, which is a daughter cell line to the leukemia cell line CCRF-CEM (CEM). Cellular ${ }^{2} \mathrm{H}_{2} \mathrm{O}$ labelling, mass spectrometry, and isotopomer analysis revealed an increase in lanosterol synthesis which was not accompanied by an increase in cholesterol flux or pool size in CEM/R2 cells. Exogenous addition of lanosterol had a negative effect on CEM/R2 and a positive effect on sensitive CEM cell viability. Treatment of CEM and CEM/R2 cells with cholesterol biosynthesis inhibitors acting on the enzymes squalene epoxidase and lanosterol synthase, both also involved in the 24,25-epoxycholesterol shunt pathway, revealed a connection of this pathway to lanosterol turnover. Our data highlight that an increased lanosterol flux
\end{abstract}

Electronic supplementary material The online version of this article (doi:10.1007/s12032-015-0717-5) contains supplementary material, which is available to authorized users.

Anders Nordström

anders.nordstrom@umu.se

1 Department of Molecular Biology, Umeå University, 90187 Umeå, Sweden

2 Department of Forest Genetics and Plant Physiology, Swedish Metabolomics Centre, Swedish University of Agricultural Sciences, Umeå, Sweden

3 Doping Laboratory, Department of Clinical Pharmacology, Karolinska University Hospital, Stockholm, Sweden

4 Department of Oncology-Pathology, Science for Life Laboratory, Karolinska Institutet, Stockholm, Sweden

5 Institute of Biochemistry, Faculty of Medicine, University of Leipzig, Leipzig, Germany poses a metabolic weakness of resistant cells that potentially could be therapeutically exploited.

Keywords Leukemia - Drug resistance Cholesterol biosynthesis $\cdot$ LC-MS $\cdot$ Stable isotope labelling mass spectrometry $\cdot$ Cancer
Abbreviations
ALL Acute lymphoblastic leukemia
CoA Coenzyme A
DNR Daunorubicin
FBS Fetal bovine serum
LC-MS Liquid chromatography-mass spectrometry
P-gp P-glycoprotein

\section{Introduction}

Cholesterol is an essential component of mammalian cell membranes and serves as a precursor for bile acids and various endocrine steroid hormones. The biosynthesis, cellular absorption, and efflux of cholesterol are tightly regulated to maintain homeostatic levels required for normal cell proliferation. A link between cholesterol and cancer was proposed over a century ago, with the discovery that tumor cells had accumulated cholesterol [1]. Since then, many studies have provided evidence for a link between carcinogenesis/tumor progression and cholesterol biosynthesis and efflux [2]. Elevated activity of hydroxymethylglutaryl-coenzyme A reductase (HMGCR), the first enzyme of the mevalonate pathway, has been shown in a range of different tumors including hepatocellular carcinoma [3], leukemia [4] and lymphoma [5]. Moreover, 
inhibition of HMGCR, the initial and rate-limiting step of cholesterol biosynthesis, with statins inhibits tumor growth in mouse xenograft models [6-8]. Epidemiological data further support a role for statins in reducing the risk of developing pancreatic cancer [9] and with an increased progression-free survival in inflammatory breast cancer [10].

Cancer is a clonal disease whereby therapeutic intervention poses a selective pressure resulting in cancer cells escaping therapy. Surviving cells are characterized by drug resistance and are often associated with disease relapse [11]. Several reports support a function of cholesterol in establishing and maintaining increased drug tolerance in cancer cells. Cholesterol has been found to be increased by $50 \%$ in isolated plasma membranes of vinblastine-resistant versus sensitive acute lymphoblastic leukemia cells (ALL) [12]. The observation that drug-resistant myeloid leukemia cell lines are more sensitive to statins than their sensitive parental lines further substantiates a role for cholesterol in chemoresistance [13]. Moreover, in vitro treatment of acute myeloid leukemia (AML) cells with chemo- or radiotherapy causes increased intracellular cholesterol levels accompanied by an increased drug tolerance, whereas inhibition of cholesterol biosynthesis with statins could restore drug sensitivity [14]. Further, it has been shown that rat and human hepatocellular carcinoma cells display increased mitochondrial cholesterol levels and HMGCR or squalene synthase (FDFT1) inhibition sensitizes those cells to mitochondria-directed chemotherapy [15]. Similarly, in a doxorubicin-resistant bladder cancer cell line, simultaneous administration of statin with doxorubicin reverted the resistant phenotype [16].

We have recently shown that resistance to daunorubicin (DNR) in an ALL cell line is associated with a rewired metabolism [17]. RNA Sequencing revealed the cholesterol biosynthetic pathway as the top canonical pathway upregulated in the resistant cells [17].

In the present study, we validate these previous findings using quantitative real-time PCR (RT-qPCR) and measure relative quantity and synthesis rates of cholesterol itself and lanosterol, the first committed intermediate in cholesterol biosynthesis, by application of ${ }^{2} \mathrm{H}_{2} \mathrm{O}$ labelling and mass spectrometry isotopomer analysis. We found that the transcriptional up-regulation of the cholesterol biosynthesis pathway does not translate into an increased cholesterol synthesis rate or quantity in the resistant cells, but rather an increased flux through the lanosterol pool. With this report we shift the focus from the importance of solely cholesterol for cancer progression and drug sensitivity to the upstream biosynthetic intermediate lanosterol. Our data reveal a previously unrecognized metabolic cost of cancer drug resistance and point toward a potential novel regulatory role of lanosterol in maintaining cholesterol homeostasis, which may be particularly critical for drug-resistant leukemia cancer cells.

\section{Materials and methods}

\section{Cell lines and growth conditions}

CCRF-CEM [CCRF CEM] (ATCC ${ }^{\circledR}$ CCL-119 ${ }^{\text {TM }}$ ) (CEM) leukemia cells were acquired through LGC Standards (Teddington, UK) from the American Type Culture Collection and maintained following the recommendations from ATCC. Detailed description of the generation of the DNR-resistant CEM/R2 is described in [17].

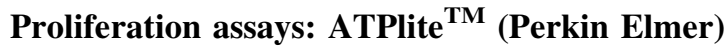

Cells were seeded in black plates with a final density of 15,000 cells/well. Simultaneously, the respective treatment was started. ATPlite ${ }^{\mathrm{TM}}$ was performed following the manufacturer's instructions. Cells were incubated for $48 \mathrm{~h}$ with or without cholesterol biosynthesis inhibitors, namely, atorvastatin $(100 \mu \mathrm{M})$, terbinafine $(25 \mu \mathrm{M})$, ketoconazole $(20 \mu \mathrm{M})$, triparanol $(2 \mu \mathrm{M})$, CI976 $(25 \mu \mathrm{M})$, all purchased from Sigma-Aldrich (MO, USA), and hymeglusin $(10 \mu \mathrm{M})$, YM-53601 $(10 \mu \mathrm{M})$ and BIBB-515 $(25 \mu \mathrm{M})$ all ordered from Santa Cruz Biotechnology (TX, USA) in the absence (vehicle control DMSO or $\mathrm{MeOH}$ ) or presence of DNR (CEM, $1 \mathrm{nM}$ and CEM/R2, $0.5 \mu \mathrm{M}$ ) in RPMI 1640 (HyClone, Fisher scientific) supplemented with $10 \%$ FBS.

Lanosterol, cholesterol, and 1,2-dimyristoyl-sn-glycero3-phosphocholine (PC), all obtained from Sigma-Aldrich (MO, USA), were dissolved in chloroform/methanol (1:1). Cholesterol or lanosterol was mixed in equimolar proportion with $\mathrm{PC}$ and dried by vacuum in a speed vacuum concentrator. The lanosterol/PC, cholesterol/PC mixture, or PC alone was re-suspended in serum-free RPMI 1640 on the day of the experiment and used within the day of preparation.

To analyze the effect of lanosterol and cholesterol, cells were incubated in serum-free RPMI 1640 medium (HyClone, Fisher scientific) for $48 \mathrm{~h}$ in the absence or presence of DNR (CEM, $100 \mathrm{nM}$ and CEM/R2, $1 \mu \mathrm{M}$ ). The negative/vehicle control always contained respective amounts of DMSO, MeOH, or PC.

\section{RNA isolation, reverse transcription, and quantitative real-time PCR}

One million cells of each CEM and CEM/R2 cells were seeded in a 6-w plate and cultured for $24 \mathrm{~h}$ in RPMI supplemented with $10 \%$ FBS without or with $50 \mu \mathrm{M}$ atorvastatin, $12.5 \mu \mathrm{M}$ terbinafine, $12.5 \mu \mathrm{M}$ BIBB515, or 
$10 \mu \mathrm{M}$ ketoconazole before harvested for RNA preparation. Total RNA isolation was performed using RNeasy ${ }^{\circledR}$ Mini Kit (Qiagen, Germany) following manufacturer's instructions. RNA $(1 \mu \mathrm{g})$ was treated with DnaseI (NEB) prior reverse transcription using iScript ${ }^{\mathrm{TM}}$ cDNA Synthesis Kit (Bio-Rad) following the manufacturer's instructions. The qPCR was set up using iTaq ${ }^{\mathrm{TM}}$ Universal $\mathrm{SYBR}^{\circledR}$ Green Supermix (Bio-Rad), and real-time PCR and data collection were performed on Bio- $\mathrm{Rad}^{\circledR} \mathrm{iQ}^{\mathrm{TM}} 5$ Real-Time PCR Detection System. Primer design procedure and detailed description of each step can be found in [18]. All qPCR primer pairs are stated in Table S1. Expression of gene-encoding proteins involved in cholesterol biosynthesis was normalized to the reference genes RPL13A, RPS18, ACTB, and GAPDH.

\section{Liquid chromatography-mass spectrometry (LC- MS) measurements}

For experiments with ${ }^{2} \mathrm{H}_{2} \mathrm{O}, 1 \times 10^{6}$ cells were cultured for $4 \mathrm{~h}$ (without or with $50 \mu \mathrm{M}$ atorvastatin, $12.5 \mu \mathrm{M}$ terbinafine, $12.5 \mu \mathrm{M}$ BIBB515 or $10 \mu \mathrm{M}$ ketoconazole) or $24 \mathrm{~h}$ (untreated comparison of lanosterol and cholesterol turnover in CEM versus CEM/R2), in RPMI 1640 medium supplemented with $10 \%$ FBS which was either diluted with sterile $\mathrm{H}_{2} \mathrm{O}$ or ${ }^{2} \mathrm{H}_{2} \mathrm{O}(99 \%)$ to a final concentration of $30 \%{ }^{2} \mathrm{H}_{2} \mathrm{O}$ (Cambridge Isotope Laboratories (MA, USA)).

The cells were centrifuged, washed at least once with PBS, transferred to an 1.5-mL Eppendorf tube and lysed/ extracted using $200 \mu \mathrm{L} 50: 50$ chloroform/methanol to which a small lab spoon of $0.2 \mu \mathrm{m}$ i.d. glass beads was added (Retsch). Tubes were placed in a Retsch Beadmill MM 400 and shaken at $30 \mathrm{~Hz}$ for 2 min. Eppendorf tubes were transferred to a centrifuge kept at $4{ }^{\circ} \mathrm{C}$ and spun at $14,000 \mathrm{rpm}$ for $10 \mathrm{~min}$ after which the supernatant was transferred to LC-MS glass vials, dried down in a speed vacuum concentrator, and stored at $-20{ }^{\circ} \mathrm{C}$ until analysis. Samples were dissolved in $20 \mu \mathrm{L}$ chloroform out of which 2-4 $\mu \mathrm{L}$ were injected into the Agilent $1290 \mathrm{LC}$ system connected to either a 6540 or 6550 Agilent Q-TOF mass spectrometer (CA, USA) and an atmospheric pressure ionization (APCI) source was used. Data were collected between $\mathrm{m} / \mathrm{z} 70$ and 1700 in positive ion mode only. The following APCI settings were used: gas temperature $200{ }^{\circ} \mathrm{C}$, vaporizer $350{ }^{\circ} \mathrm{C}$, gas flow $11 \mathrm{l} / \mathrm{min}$, nebulizer pressure $40 \mathrm{psig}$, Vcap 3500, corona 4, fragmentor 100 , Skimmer1 45, and OctapoleRFPeak 750. All samples were separated using reverse phase only, Kinetex C18, $100 \mathrm{~mm} \times 2.1 \mathrm{~mm}, 2.6 \mu \mathrm{M} 100 \AA$, Phenomenex (CA, USA). For elution, solvents reversed phase (A) $\mathrm{H}_{2} \mathrm{O}, 0.1 \%$ formic acid (B) 75:25 methanol/isopropanol, $0.1 \%$ formic acid were used. All solvents were of HPLC grade. Linear gradients were used for all separations and were devised as follows for reversed phase separation $(0.5 \mathrm{~mL} / \mathrm{min}) \min 0$ : $5 \% \mathrm{~B}, \min 8: 95 \% \mathrm{~B}, \min 10: 95 \% \mathrm{~B}, \min 10.2: 5 \% \mathrm{~B}$, min 12: $5 \%$ B. Raw data were processed and analyzed using MassHunter Qual, Agilent (CA, USA). Identification of metabolites in all experiments was carried out using synthetic standards obtained from Sigma-Aldrich (MO, USA) and Inventia Pty. Ltd (NSW, Australia) comparing accurate mass, retention time, and in some cases MS/MS spectra.

\section{Results}

\section{The cholesterol biosynthetic pathway is up-} regulated in DNR-resistant CEM/R2 cells

Levels of gene expression for all cholesterol biosynthetic genes in CEM and CEM/R2 were monitored using RTqPCR (Fig. 1a, b). Interestingly, mRNA levels of HMGCR are significantly lower in CEM/R2 cells (Fig. 1b), whereas mRNA levels of all other enzymes involved in cholesterol biosynthesis except five are significantly up-regulated (Fig. 1b). The highest fold change in mRNA expression was obtained for CYP51A1 (cytochrome P450, family 51, subfamily A, polypeptide 1) and ABCA1 with approximately fivefold higher mRNA levels in CEM/R2 when compared to CEM cells followed by squalene epoxidase (SQLE) with approximately threefold higher mRNA levels in CEM/R2 cells (Fig. 1b).

\section{Increased flux through the lanosterol but not the cholesterol pool in resistant CEM/R2 cells}

Next, we measured relative quantity of lanosterol, the first cholesterol biosynthesis intermediate committed solely to the cholesterol pathway, and cholesterol using liquid chromatography-mass spectrometry (LC-MS). Surprisingly, we found that in spite of a transcriptionally up-regulated cholesterol pathway in CEM/R2 cells, the relative concentration of lanosterol and cholesterol was lower in CEM/R2 cells (Fig. 2a). This observation led us to analyze whether the lower relative quantity of lanosterol and cholesterol can be explained by lower synthesis rate in $\mathrm{CEM} / \mathrm{R} 2$ cells. Thus, we set out to measure de novo synthesis of cholesterol itself and lanosterol using ${ }^{2} \mathrm{H}_{2} \mathrm{O}$ labelling and LC-MS [19]. By growing cells in media diluted with ${ }^{2} \mathrm{H}_{2} \mathrm{O}$, the stable isotope ${ }^{2} \mathrm{H}$ will be incorporated throughout the cellular metabolism which can be followed in the individual metabolites by mass spectrometry and isotopomer analysis, illustrated in Fig. 2b. Virtually, no de novo formation of cholesterol could be observed 


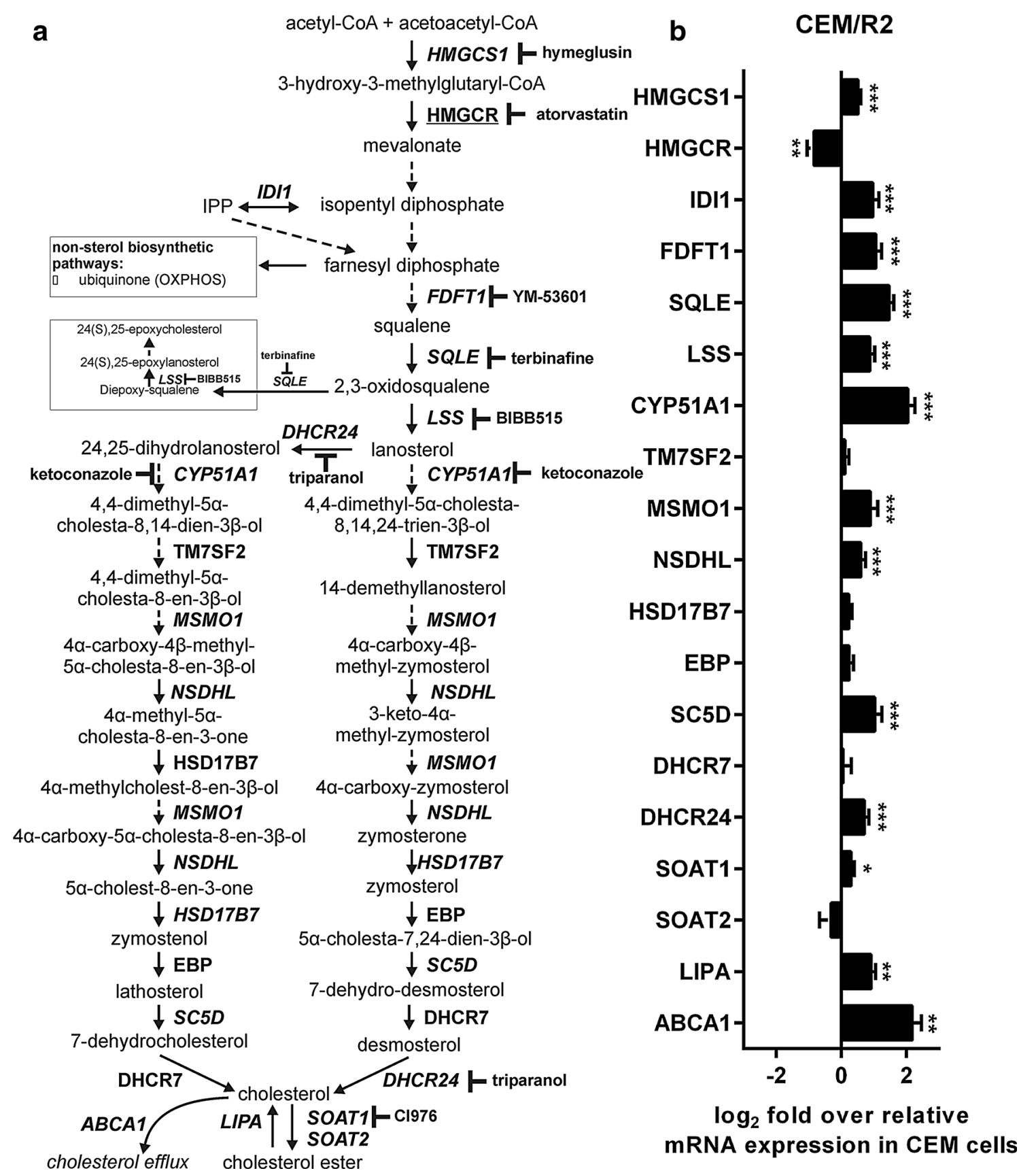

Fig. 1 Cholesterol biosynthesis pathway is altered in CEM/R2 cells. a Outlined cholesterol synthesis pathway in which all genes with increased mRNA expression are highlighted in green and HMGCR as its mRNA expression level is lower in CEM/R2 cells is highlighted in red. Major points of inhibition by cholesterol biosynthesis inhibitors are highlighted in red. b mRNA expression levels of all genes

(Fig. 2b, c). Lanosterol on the other hand which displayed a lower relative concentration in CEM/R2 cells compared to CEM (Fig. 2a) exhibited at the same time a higher relative ${ }^{2} \mathrm{H}$ incorporation in CEM/R2 cells, suggesting a higher flux through the lanosterol pool in the resistant cells (Fig. 2c). involved in the cholesterol biosynthetic pathway is shown as $\log 2$ fold over relative mRNA expression for each gene in CEM cells. Significance was assessed using a two-tailed unpaired $t$ test. Data are shown as mean \pm SEM of six independent experiments. $* P \leq 0.05 ; * * P \leq 0.01 ; * * * P \leq 0.001$

Exogenous addition of lanosterol is beneficial for drug-sensitive CEM but disadvantageous for resistant $\mathbf{C E M} / \mathbf{R} 2$ cells

With no apparent transfer of the ${ }^{2} \mathrm{H}$ label from lanosterol to cholesterol (Fig. 2b), it is reasonable to assume that in 

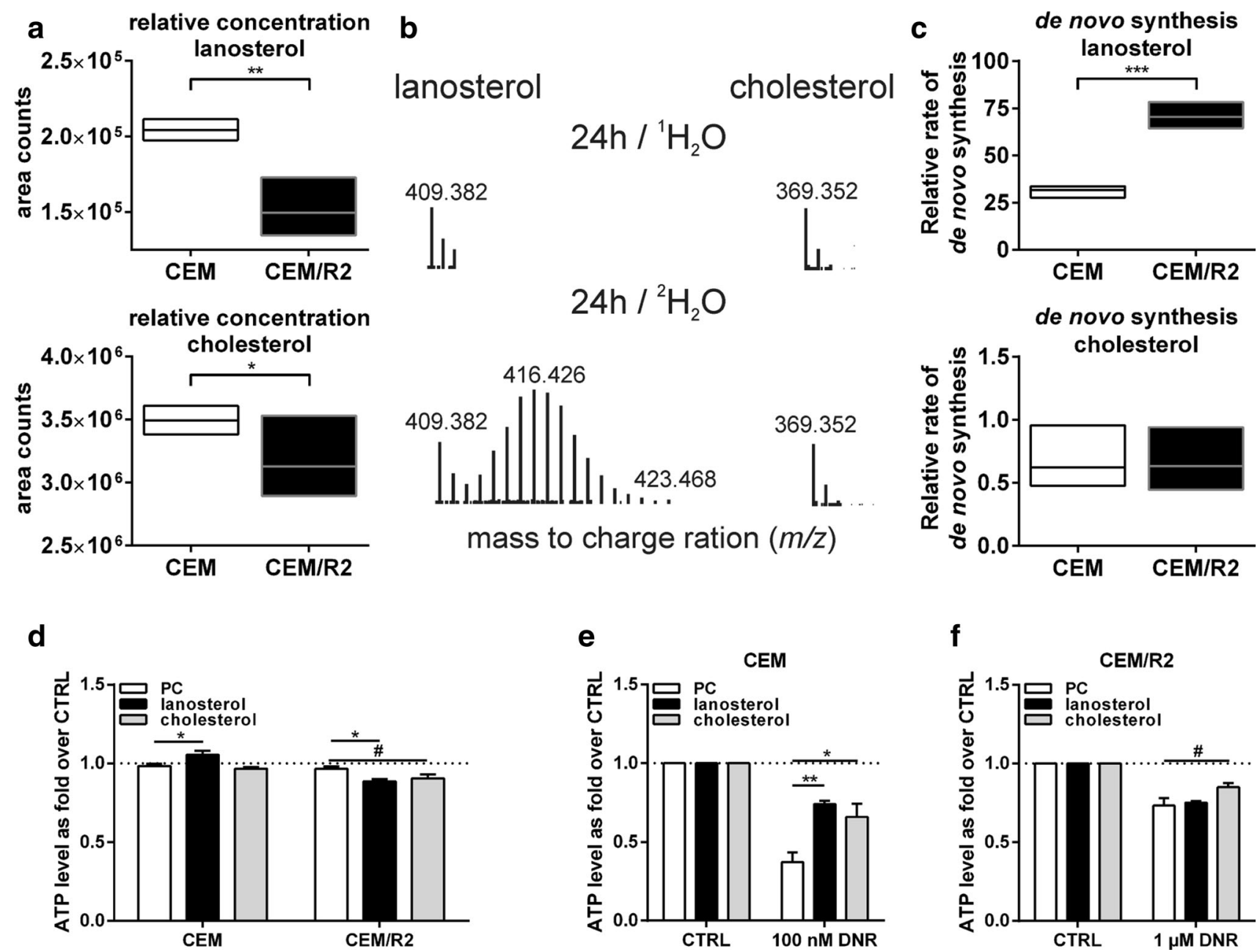

Fig. 2 Resistant leukemia cells CEM/R2 exhibit an increased flux through the lanosterol but not the cholesterol pool and are negatively affected by exogenous lanosterol addition. a Relative concentration of lanosterol and cholesterol in CEM versus CEM/R2 cells as determined by LC-MS. b Mass spectra of lanosterol (left) and cholesterol (right) after cell growth for $24 \mathrm{~h}$ using regular media (top) and media with addition of $30 \%{ }^{2} \mathrm{H}_{2} \mathrm{O}$ (bottom). c Data showing de novo synthesis of lanosterol and cholesterol measured on cells grown in $30 \%{ }^{2} \mathrm{H}_{2} \mathrm{O}$. a-c Data of a single experiment carried out in five replicates are shown as minimum to maximum with line at mean. d Viability of CEM and CEM/R2 cells $(n=4)$ that were grown for

$\mathrm{CEM} / \mathrm{R} 2$ cells the increased lanosterol production reflects that lanosterol, rather than just being an intermediate in the cholesterol biosynthesis either is exported out of the cells or fills another function. Thus, to probe whether increased lanosterol flux is essential to maintain resistance or rather a metabolic consequence of resistance, we investigated the influence of exogenous addition of lanosterol and cholesterol on CEM and CEM/R2 cell viability (Fig. 2d). Lanosterol addition was beneficial for CEM but disadvantageous for CEM/R2 cell viability (Fig. 2d). Cholesterol had no apparent effect on CEM cells and was slightly disadvantageous for CEM/R2 cells
$48 \mathrm{~h}$ in serum-free RPMI 1640 in presence of $50 \mu \mathrm{M}$ 1,2-dimyristoylsn-glycero-3-phosphocholine (PC), lanosterol/PC mixture (each $25 \mu \mathrm{M}$ ), or cholesterol/PC mixture (each $25 \mu \mathrm{M}$ ). Viability of CEM (e) and CEM/R2 (f) cells that were incubated for $48 \mathrm{~h}$ in absence or

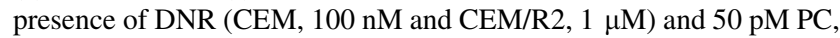
lanosterol/PC mixture (each $25 \mu \mathrm{M}$ ), or cholesterol/PC mixture (each $25 \mu \mathrm{M})$. d-f Data are shown as mean \pm SEM of four independent experiments carried out in triplicate, $P$ values were determined using an ordinary one-way ANOVA with Dunnett's multiple comparisons test. ${ }^{\#} \leq 0.1 ; * P \leq 0.05 ; * * P \leq 0.0110$

(Fig. 2d). Next, we evaluated the pro-survival effect of lanosterol and cholesterol on DNR sensitivity of CEM and CEM/R2 cells (Fig. 2e, f). Presence of both lanosterol and cholesterol decreased the sensitivity of CEM cells to DNR (Fig. 2e), thus supporting a pro-survival effect of lanosterol for cancer cells. However, in CEM/R2 cells, exogenous lanosterol addition did not trigger such an effect and cholesterol addition decreased sensitivity to DNR only slightly (Fig. 2f). We conclude therefore that the increased lanosterol flux represents a metabolic cost rather than a survival advantage for the resistant CEM/R2 cells. 


\section{Differential sensitivity of CEM versus CEM/R2 cells toward cholesterol biosynthesis inhibitors}

To gain further insights into the differences of the cholesterol biosynthetic pathway between sensitive and resistant leukemia cells, with special focus on both rate-limiting steps and steps producing or consuming lanosterol, we evaluated the potential of different cholesterol biosynthesis inhibitors as both cytostatic agents (Fig. 3a) and positive modulators of drug sensitivity (Figure S1) in CEM and CEM/R2 cells.

If cholesterol lowering per se has an anticancer effect, the transcriptional up-regulation of the cholesterol biosynthesis pathway in CEM/R2 cells points toward an increased relevance of this pathway in resistant cells. Therefore, one would expect that all inhibitors of cholesterol biosynthesis, administered alone, would have a stronger effect on CEM/R2 cell viability. This did not turn out to be the case. However, terbinafine, an antifungal compound inhibiting SQLE, triparanol, an inhibitor of 24-dehydrocholesterol reductase (DHCR24), both shown to suppress tumor growth [20-23], as well as CI976, a potent and selective Acyl-CoA/cholesterol acyltransferase (SOAT1) inhibitor [24, 25] did affect CEM/R2 more than the sensitive CEM cells (Fig. 2a). a

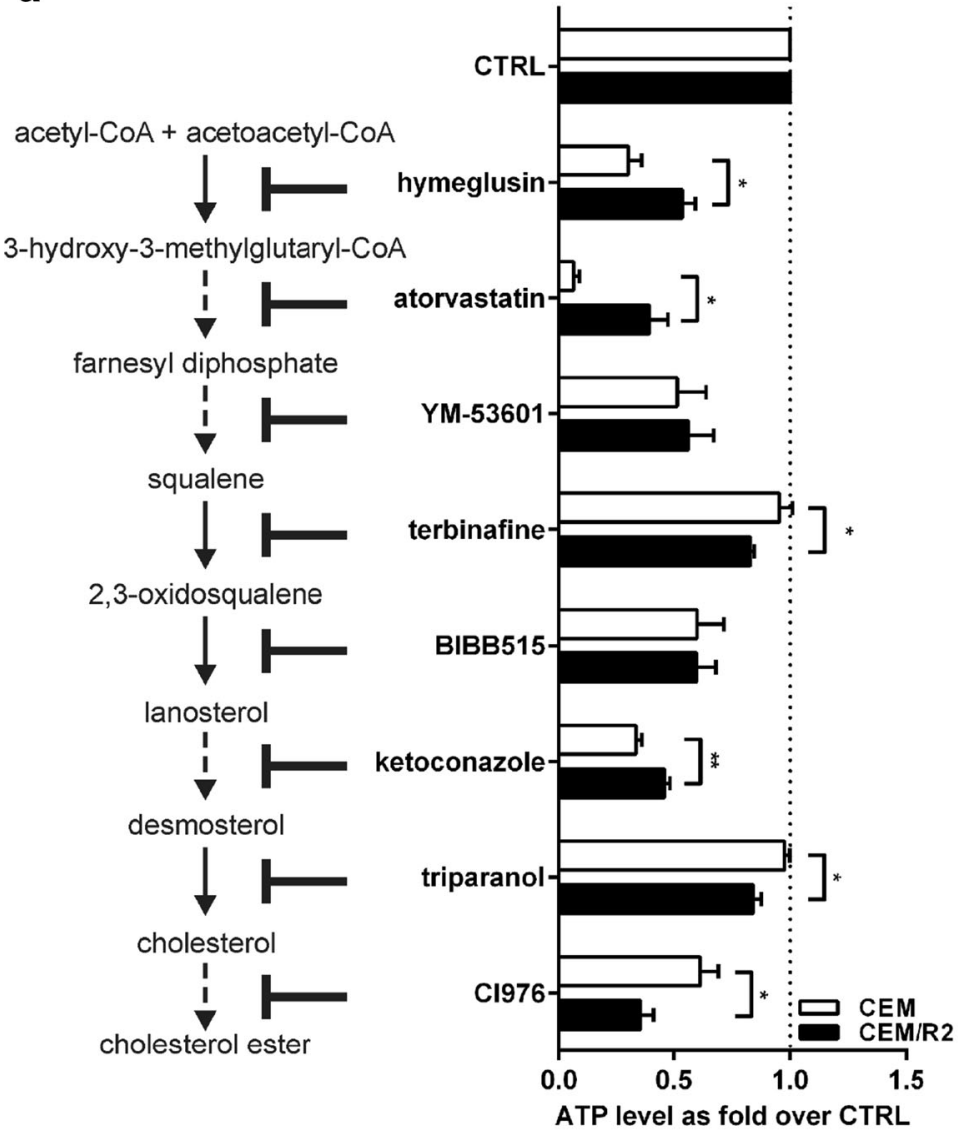

b

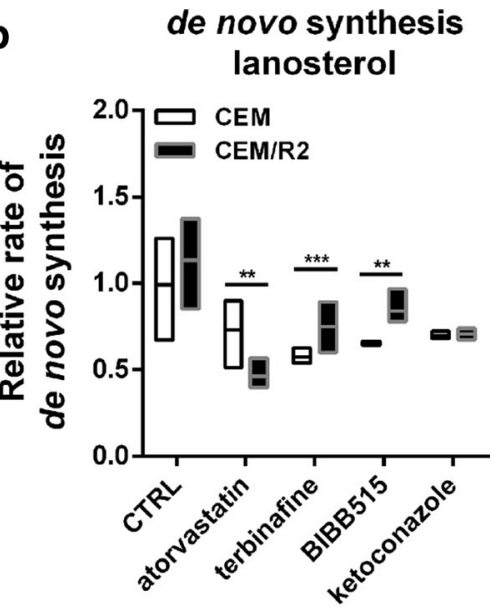

C

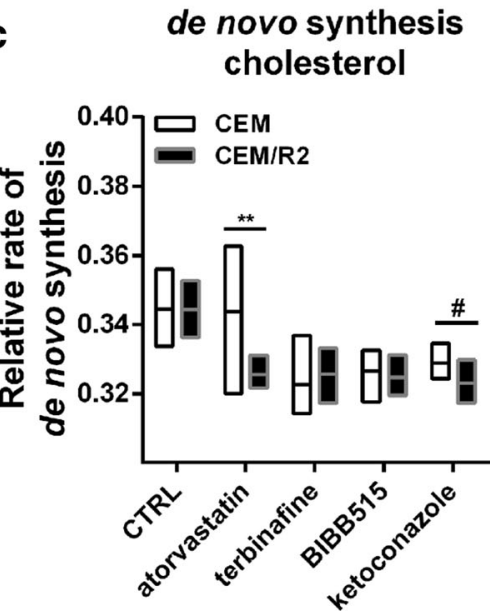

Fig. 3 Effect of cholesterol biosynthesis inhibitors on cell viability and lanosterol as well as cholesterol relative synthesis rate of CEM and CEM/R2 cells. a CEM and CEM/R2 cells were incubated for $48 \mathrm{~h}$ with $10 \mu \mathrm{M}$ hymeglusin, $100 \mu \mathrm{M}$ atorvastatin, $10 \mu \mathrm{M} \mathrm{YM}-$ 53601, $25 \mu \mathrm{M}$ terbinafine, $25 \mu \mathrm{M}$ BIBB-515,10 $\mu \mathrm{M}$ ketoconazole, $2 \mu \mathrm{M}$ triparanol, or $25 \mu \mathrm{M}$ CI976 in RPMI 1640 supplemented with $10 \%$ FBS in comparison with vehicle control DMSO or $\mathrm{MeOH}$. Hymeglusin, atorvastatin, and ketoconazole had a stronger effect on CEM cells, whereas terbinafine, triparanol, and CI976 were more effective on CEM/R2 cells, and no difference in sensitivity between CEM and CEM/R2 cells was observed for YM-53601 and BIBB-515.
Data are shown as mean \pm SEM of three independent experiments carried out in triplicates. Relative synthesis rate of lanosterol (b) and cholesterol (c) in CEM and CEM/R2 cells as determined by LC-MS after cells were cultured for $4 \mathrm{~h}$ in presence or absence of $50 \mu \mathrm{M}$ atorvastatin or $12.5 \mu \mathrm{M}$ terbinafine, $12.5 \mu \mathrm{M}$ BIBB515 or $10 \mu \mathrm{M}$ ketoconazole, in RPMI 1640 supplemented with $10 \%$ FBS and $30 \%$ ${ }^{2} \mathrm{H}_{2} \mathrm{O}$. Data of a single experiment carried out in four replicates are shown as minimum to maximum with line at mean. a-c Significance was assessed using a two-tailed unpaired $t$ test ${ }^{\#} \leq 0.1 ; * P \leq 0.05$; $* * P \leq 0.01 ; * * * P \leq 0.001$ 
Lanosterol de novo synthesis rate correlates with sensitivity toward cholesterol biosynthesis inhibitors

If the increased lanosterol flux is a metabolic burden for the resistant cells, while being associated with increased viability in sensitive cells, one could argue that cholesterol synthesis inhibitors inducing a relatively larger reduction in lanosterol flux in resistant compared to sensitive cells should result in lower viability of sensitive CEM to CEM/ $\mathrm{R} 2$ cells or vice versa. To test this hypothesis, we analyzed the effect on de novo synthesis of lanosterol and cholesterol when exposed to atorvastatin acting at HMGCR, the first rate-limiting enzyme, terbinafine, acting at the proposed second rate-limiting step SQLE, BIBB-515, which inhibits lanosterol synthase (LSS), as well as ketoconazole, an inhibitor of the CYP51A1 [26, 27] directly downstream of lanosterol (Fig. 3b, c). All four inhibitors reduced the de novo synthesis rate of lanosterol and cholesterol (except of atorvastatin in CEM cells) significantly in both cell lines when compared to control (Fig. 3b, c). Next, we compared the relative degree of reduction of lanosterol de novo synthesis for sensitive versus resistant cells. We observed that atorvastatin reduced the de novo synthesis through the lanosterol pool more in resistant cells, whereas the opposite was true for terbinafine and BIBB515 (Fig. 3b). No difference in lanosterol biosynthesis rate comparing CEM and CEM/R2 cells was observed when cells were treated with ketoconazole (Fig. 3b). The comparison of the viability data obtained for atorvastatin and terbinafine (Fig. 3a) with the lanosterol flux information gained by LC-MS (Fig. 3b) reveals a connection between sensitivity to those inhibitors and their effect on lanosterol de novo synthesis rate.

\section{Inhibition of the 24,25-epoxycholesterol shunt pathway suggests its differential regulation in resistant cells}

The resistant cells show an increased flux of lanosterol which is disadvantageous for them. It is reasonable to assume that these cells have regulatory mechanisms that are different when compared to sensitive cells. These mechanisms can either be the cause of or are required to manage the observed increased lanosterol flux. One such regulatory mechanism of cholesterol biosynthesis is the 24,25-epoxycholesterol (24,25-EC) shunt pathway (Figs. 1a, 4a). 24,25EC has been shown to cause a decreased cholesterol synthesis rate by inhibition of HMGCR [28] and DHCR24 [29] and thus prevents accumulation of newly synthesized cholesterol which could cause ER stress and cell toxicity $[30,31]$. Since the inhibitors of this shunt pathway, namely terbinafine inhibiting SQLE and BIBB515 inhibiting LSS, had both reduced de novo synthesis of lanosterol more in CEM/R2 cells, we evaluated their effect on mRNA level of genes involved in the cholesterol biosynthetic pathway, in comparison with the effects induced by atorvastatin and ketoconazole treatment (Fig. 4b, Figure S2).

Both BIBB515 and terbinafine but not atorvastatin and ketoconazole induce differential expression of HMGCR, SQLE, and DHCR24 when comparing sensitive and resistant CEM cells (Fig. 4b). Several other genes that are involved in the main cholesterol biosynthesis pathway display the same pattern (Figure S2). The inhibition of the 24,25-EC shunt pathway, but not the main cholesterol biosynthesis pathway, produces an up-regulation of ratelimiting steps of the cholesterol biosynthetic pathway specific to resistant cells (Fig. 4b). We are therefore suggesting that either the shunt pathway is critical for resistant cells to regulate an increased lanosterol flux or a differentially regulated shunt pathway is the cause for the increased lanosterol flux.

\section{Discussion}

An altered cholesterol metabolism is critical for rapidly proliferating cancer cells and plays a role in development of resistance. Based on our findings, we suggest that lanosterol, the first intermediate committed solely toward cholesterol biosynthesis, is a valuable marker to detect alterations of the cellular cholesterol homeostasis. We show that the increased lanosterol flux in a DNR-resistant daughter cell line of the T-ALL leukemia cell line CEM represents a metabolic cost that can potentially have therapeutic implications. In a broader sense, our results highlight that phenotyping cancers with respect to cholesterol metabolism can be useful for therapy guidance.

We used RT-qPCR and compared mRNA levels of all proteins involved in the cholesterol biosynthesis pathway (Fig. 1a) between DNR-sensitive CEM cells and the resistant daughter cell line CEM/R2. The almost uniformly increased expression in the resistant cells (Fig. 1b) was not matched by increased levels of cholesterol or lanosterol (Fig. 2a). Using ${ }^{2} \mathrm{H}_{2} \mathrm{O}$ labelling of cultured cells, we could demonstrate an increased biosynthetic flux of lanosterol (Fig. 2b, c) with no concomitant accumulation of lanosterol or cholesterol (Fig. 2a). It is therefore reasonable to assume that in CEM/R2 cells the increased lanosterol production reflects that lanosterol, rather than just being an intermediate in the cholesterol biosynthesis, is exported out of the cell [32-34] or fills another function. Membrane associated lanosterol will alter plasma membrane organization relative to cholesterol [35], which potentially could impact drug tolerance [12]. Further, lanosterol has been proposed to act as a survival factor for dopaminergic 


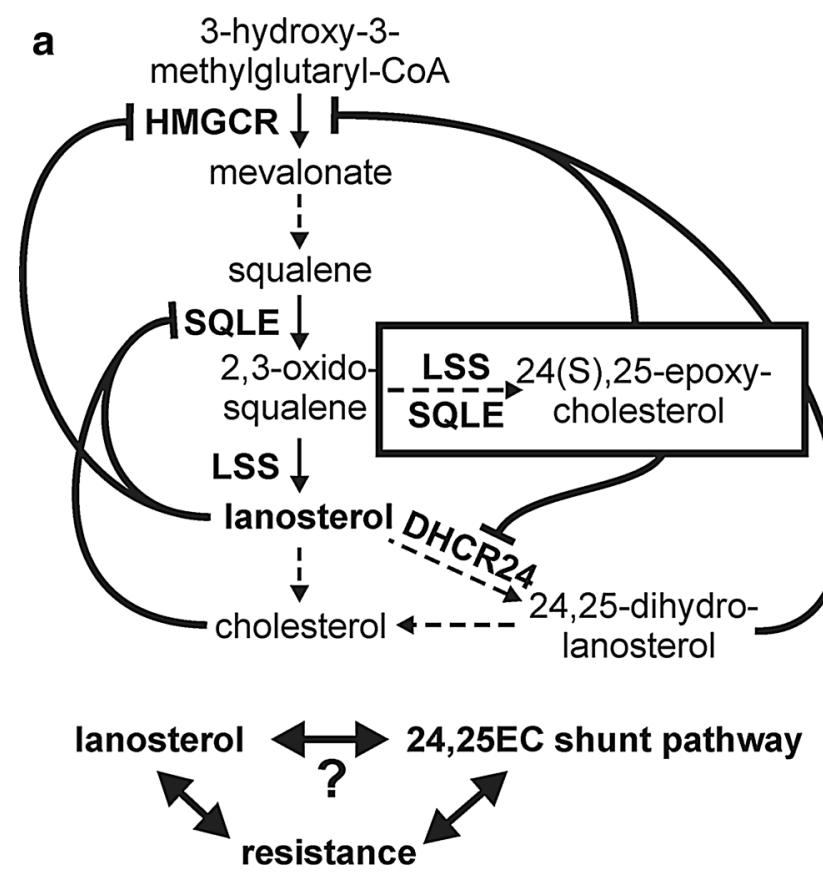

Fig. 4 Differentially regulated 24,25-EC shunt pathway in CEM/R2 cells potentially cause or effect of increased lanosterol flux. a HMGCR, the first rate-limiting step of cholesterol biosynthesis is negatively regulated by lanosterol, 24,25-dihydrolanosterol and 24,25-EC [50]. SQLE is regulated by lanosterol and cholesterol [41-45] and participates, together with LSS, in a shunt of the mevalonate pathway that produces 24,25-EC. 24,25-EC itself inhibits HMGCR [46] and DHCR24 [47]. b BIBB515 and terbinafine, acting at LSS and SQLE, respectively, induce differential expression of

neurons, potentially via a mitochondrial decoupling mechanism [36]. We observed that exogenously applied lanosterol negatively affected viability of resistant cell but had a positive effect on cell viability of DNR-sensitive CEM cells. Moreover, since lanosterol has been described as a survival factor [36], we tested the effect of lanosterol on DNR sensitivity of both CEM and CEM/R2 by co-administration of DNR and lanosterol, which revealed that lanosterol presence decreased DNR sensitivity of CEM cells but had no such effect on CEM/R2 cells. We conclude from the results from all those experiments that the increased lanosterol flux is a stressor for the resistant cells and thus a negative consequence of the resistance. Consequently, it is reasonable to hypothesize that resistant cells have different regulatory mechanisms of the cholesterol biosynthetic pathway to cope with the increased lanosterol flux. Inhibition of different steps of the cholesterol pathway revealed differential effects on viability when comparing sensitive and resistant cells (Fig. 3a). Further, using ${ }^{2} \mathrm{H}_{2} \mathrm{O}$ labelling mass spectrometry, we show that inhibitors that reduce the lanosterol synthesis rate more in the resistant cells affected their viability less upon treatment with the respective inhibitors (Fig. 3b, c). b
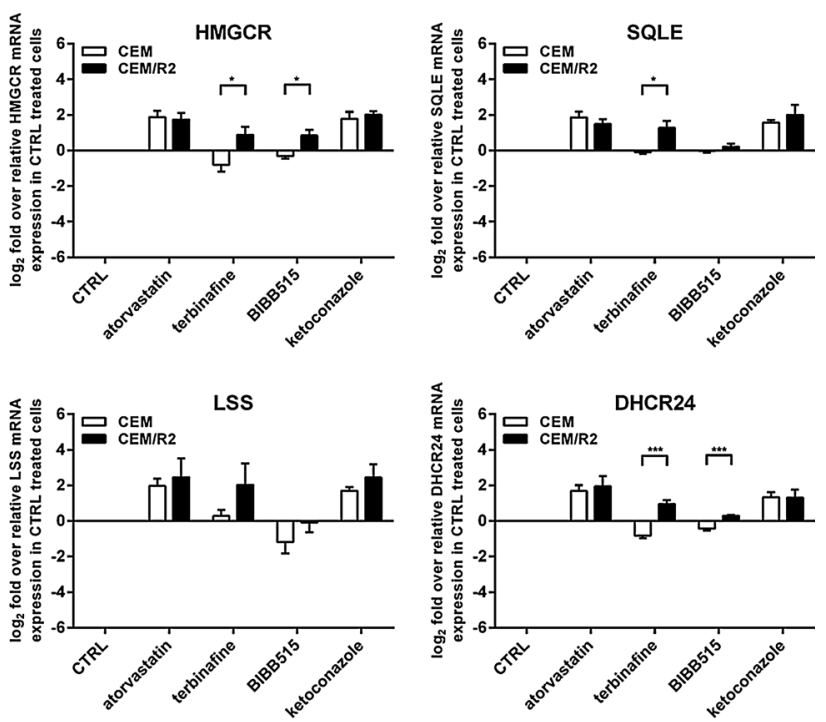

HMGCR, SQLE, and DHCR between CEM and CEM/R2. Cells were cultured for $24 \mathrm{~h}$ in presence or absence of $50 \mu \mathrm{M}$ atorvastatin, $12.5 \mu \mathrm{M}$ terbinafine, $12.5 \mu \mathrm{M}$ BIBB515, or $10 \mu \mathrm{M}$ ketoconazole in RPMI 1640 supplemented with $10 \%$ FBS. mRNA expression levels are shown as $\log 2$ relative mRNA expression fold over vehicle-treated $\mathrm{CEM}$ or CEM/R2 cells, respectively. Data are shown as mean $\pm \mathrm{SEM}$ of four independent experiments, $P$ values were determined using an unpaired $t$ test. $* P \leq 0.05 ; * * P \leq 0.01 ; * * * P \leq 0.001$

Lanosterol itself has been shown to be involved in posttranslational regulation of the cholesterol biosynthesis pathway through induction of proteasomal degradation of HMGCR [37] and SQLE [38]. The enzyme SQLE, which is inhibited by terbinafine, is further transcriptionally [39, 40] and posttranslationally regulated by cholesterol [41, 42] and participates, together with LSS, which can be inhibited by BIBB515, in a shunt of the mevalonate pathway that produces 24,25-EC (Figs. 1a, 4a). By measuring gene expression of all genes in the cholesterol biosynthesis when applying one of the inhibitors, atorvastatin, terbinafine, BIBB515, or ketoconazole, a pattern emerged. Inhibitors of SQLE or LSS, both of which are enzymes involved in the main pathway and in the 24,25-EC shunt pathway, resulted in a reciprocal expression pattern for many genes when comparing CEM versus CEM/R2. In contrast, atorvastatin and ketoconazole that do not affect the 24,25-EC shunt pathway exert the same effects on mRNA levels of cholesterol biosynthesis genes in both sensitive and resistant cells (Figs. 4b, S2).

In conclusion, our data provide a novel connection between drug resistance and increased lanosterol flux and also links the 24,25-EC shunt pathway with resistance. We 
believe that there is a high potential for exploitation of this knowledge in personalized therapy guidance.

Acknowledgments This research was supported by grants from the Swedish Foundation for Strategic Research (to A. N.), the Swedish Research Council 2007-5143 (to A. N.), the Erling-Persson Family Foundation (to Umeå University), and Jane and Dan Olsson Foundation (to A. N.).

\section{Compliance with ethical standards}

\section{Conflict of interest None.}

Open Access This article is distributed under the terms of the Creative Commons Attribution 4.0 International License (http://crea tivecommons.org/licenses/by/4.0/), which permits unrestricted use, distribution, and reproduction in any medium, provided you give appropriate credit to the original author(s) and the source, provide a link to the Creative Commons license, and indicate if changes were made.

\section{References}

1. White CP. On the occurrence of crystals in tumours. J Pathol Bacteriol. 1909;13(1):3-10. doi:10.1002/path.1700130103.

2. Cruz PM, Mo H, McConathy WJ, Sabnis N, Lacko AG. The role of cholesterol metabolism and cholesterol transport in carcinogenesis: a review of scientific findings, relevant to future cancer therapeutics. Front Pharmacol. 2013;4:119. doi:10.3389/fphar. 2013.00119.

3. Kawata S, Takaishi K, Nagase T, Ito N, Matsuda Y, Tamura S, et al. Increase in the active form of 3-hydroxy-3-methylglutaryl coenzyme A reductase in human hepatocellular carcinoma: possible mechanism for alteration of cholesterol biosynthesis. Cancer Res. 1990;50(11):3270-3.

4. Vitols S, Norgren S, Juliusson G, Tatidis L, Luthman H. Multilevel regulation of low-density lipoprotein receptor and 3-hydroxy-3-methylglutaryl coenzyme A reductase gene expression in normal and leukemic cells. Blood. 1994;84(8):2689-98.

5. Harwood HJ Jr, Alvarez IM, Noyes WD, Stacpoole PW. In vivo regulation of human leukocyte 3-hydroxy-3-methylglutaryl coenzyme A reductase: increased enzyme protein concentration and catalytic efficiency in human leukemia and lymphoma. J Lipid Res. 1991;32(8):1237-52.

6. Gao J, Jia WD, Li JS, Wang W, Xu GL, Ma JL, et al. Combined inhibitory effects of celecoxib and fluvastatin on the growth of human hepatocellular carcinoma xenografts in nude mice. J Int Med Res. 2010;38(4):1413-27.

7. Huang EH, Johnson LA, Eaton K, Hynes MJ, Carpentino JE, Higgins PD. Atorvastatin induces apoptosis in vitro and slows growth of tumor xenografts but not polyp formation in MIN mice. Dig Dis Sci. 2010;55(11):3086-94. doi:10.1007/s10620-010-1157-x.

8. Huang H, Cui XX, Chen S, Goodin S, Liu Y, He Y, et al. Combination of lipitor and celebrex inhibits prostate cancer VCaP cells in vitro and in vivo. Anticancer Res. 2014;34(7):3357-63.

9. Khurana V, Sheth A, Caldito G, Barkin JS. Statins reduce the risk of pancreatic cancer in humans-a case-control study of half a million veterans. Pancreas. 2007;34(2):260-5. doi:10.1097/Mpa. 0b013e318030e963.

10. Brewer TM, Masuda H, Liu DD, Shen Y, Liu P, Iwamoto T, et al. Statin use in primary inflammatory breast cancer: a cohort study. Br J Cancer. 2013;109(2):318-24. doi:10.1038/Bjc.2013.342.
11. Haffner MC, Mosbruger T, Esopi DM, Fedor H, Heaphy CM, Walker DA, et al. Tracking the clonal origin of lethal prostate cancer. J Clin Investig. 2013;123(11):4918-22. doi:10.1172/JCI70354.

12. May GL, Wright LC, Dyne M, Mackinnon WB, Fox RM, Mountford CE. Plasma membrane lipid composition of vinblastine sensitive and resistant human leukaemic lymphoblasts. Int J Cancer. 1988;42(5):728-33.

13. Maksumova L, Ohnishi K, Muratkhodjaev F, Zhang W, Pan L, Takeshita A, et al. Increased sensitivity of multidrug-resistant myeloid leukemia cell lines to lovastatin. Leukemia. 2000;14(8):1444-50.

14. Li HY, Appelbaum FR, Willman CL, Zager RA, Banker DE. Cholesterol-modulating agents kill acute myeloid leukemia cells and sensitize them to therapeutics by blocking adaptive cholesterol responses. Blood. 2003;101(9):3628-34. doi:10.1182/blood2002-07-2283.

15. Montero J, Morales A, Llacuna L, Lluis JM, Terrones O, Basanez G, et al. Mitochondrial cholesterol contributes to chemotherapy resistance in hepatocellular carcinoma. Cancer Res. 2008; 68(13):5246-56. doi:10.1158/0008-5472.CAN-07-6161.

16. Greife A, Tukova J, Steinhoff C, Scott SD, Schulz WA, Hatina J. Establishment and characterization of a bladder cancer cell line with enhanced doxorubicin resistance by mevalonate pathway activation. Tumour Biol. 2015;36(5):3293-300. doi:10.1007/ s13277-014-2959-9.

17. Staubert $\mathrm{C}$, Bhuiyan $\mathrm{H}$, Lindahl A, Broom OJ, Zhu Y, Islam S, et al. Rewired metabolism in drug-resistant leukemia cells: a metabolic switch hallmarked by reduced dependence on exogenous glutamine. J Biol Chem. 2015;290(13):8348-59. doi:10. 1074/jbc.M114.618769.

18. Staubert C, Broom OJ, Nordstrom A. Hydroxycarboxylic acid receptors are essential for breast cancer cells to control their lipid/fatty acid metabolism. Oncotarget. 2015;6(23):19706-20.

19. Castro-Perez J, Previs SF, McLaren DG, Shah V, Herath K, Bhat $\mathrm{G}$, et al. In vivo D2O labeling to quantify static and dynamic changes in cholesterol and cholesterol esters by high resolution LC/MS. J Lipid Res. 2011;52(1):159-69. doi:10.1194/jlr.D009787.

20. Lee WS, Chen RJ, Wang YJ, Tseng H, Jeng JH, Lin SY, et al. In vitro and in vivo studies of the anticancer action of terbinafine in human cancer cell lines: G0/G1 p53-associated cell cycle arrest. Int J Cancer. 2003;106(1):125-37. doi:10.1002/ijc.11194.

21. Petranyi G, Ryder NS, Stutz A. Allylamine derivatives: new class of synthetic antifungal agents inhibiting fungal squalene epoxidase. Science. 1984;224(4654):1239-41.

22. Chien MH, Lee TS, Kao C, Yang SF, Lee WS. Terbinafine inhibits oral squamous cell carcinoma growth through anti-cancer cell proliferation and anti-angiogenesis. Mol Carcinog. 2012; 51(5):389-99. doi:10.1002/Mc.20800.

23. Bi X, Han X, Zhang F, He M, Zhang Y, Zhi XY, et al. Triparanol suppresses human tumor growth in vitro and in vivo. Biochem Biophys Res Commun. 2012;425(3):613-8. doi:10.1016/j.bbrc. 2012.07.136.

24. Field FJ, Albright E, Mathur S. Inhibition of acylcoenzyme A: cholesterol acyltransferase activity by PD128O42: effect on cholesterol metabolism and secretion in CaCo-2 cells. Lipids. 1991;26(1):1-8.

25. O'Brien PM, Sliskovic DR, Blankley CJ, Roth BD, Wilson MW, Hamelehle KL, et al. Inhibitors of acyl-CoA:cholesterol O-acyl transferase (ACAT) as hypocholesterolemic agents. 8. Incorporation of amide or amine functionalities into a series of disubstituted ureas and carbamates. Effects on ACAT inhibition in vitro and efficacy in vivo. J Med Chem. 1994;37(12):1810-22.

26. Gupta S, Kim JM, Gollapudi S. Reversal of daunorubicin resistance in P388/Adr cells by itraconazole. J Clin Investig. 1991;87(4):1467-9. doi:10.1172/Jci115154.

27. Vreugdenhil G, Raemaekers JM, van Dijke BJ, de Pauw BE. Itraconazole and multidrug resistance: possible effects on 
remission rate and disease-free survival in acute leukemia. Ann Hematol. 1993;67(3):107-9.

28. Gardner RG, Shan H, Matsuda SP, Hampton RY. An oxysterolderived positive signal for 3-hydroxy- 3-methylglutaryl-CoA reductase degradation in yeast. J Biol Chem. 2001;276(12): 8681-94. doi:10.1074/jbc.M007888200.

29. Zerenturk EJ, Kristiana I, Gill S, Brown AJ. The endogenous regulator 24(S),25-epoxycholesterol inhibits cholesterol synthesis at DHCR24 (Seladin-1). Biochim Biophys Acta. 2012;1821(9): 1269-77. doi:10.1016/j.bbalip.2011.11.009.

30. Brown AJ. 24(S),25-epoxycholesterol: a messenger for cholesterol homeostasis. Int J Biochem Cell Biol. 2009;41(4):744-7. doi:10.1016/j.biocel.2008.05.029.

31. Wong J, Quinn CM, Gelissen IC, Brown AJ. Endogenous 24(S),25-epoxycholesterol fine-tunes acute control of cellular cholesterol homeostasis. J Biol Chem. 2008;283(2):700-7. doi:10.1074/jbc.M706416200.

32. Echevarria F, Norton RA, Nes WD, Lange Y. Zymosterol is located in the plasma-membrane of cultured human fibroblasts. J Biol Chem. 1990;265(15):8484-9.

33. Johnson WJ, Fischer RT, Phillips MC, Rothblat GH. Efflux of newly synthesized cholesterol and biosynthetic sterol intermediates from cells-dependence on acceptor type and on enrichment of cells with cholesterol. J Biol Chem. 1995;270(42):25037-46.

34. Wang N, Yvan-Charvet L, Lutjohann D, Mulder M, Vanmierlo T, Kim TW, et al. ATP-binding cassette transporters G1 and G4 mediate cholesterol and desmosterol efflux to HDL and regulate sterol accumulation in the brain. FASEB J. 2008;22(4):1073-82. doi:10.1096/fj.07-9944com.

35. Cournia Z, Ullmann GM, Smith JC. Differential effects of cholesterol, ergosterol and lanosterol on a dipalmitoyl phosphatidylcholine membrane: a molecular dynamics simulation study. J Phys Chem B. 2007;111(7):1786-801. doi:10.1021/jp065172i.

36. Lim L, Jackson-Lewis V, Wong LC, Shui GH, Goh AX, Kesavapany S, et al. Lanosterol induces mitochondrial uncoupling and protects dopaminergic neurons from cell death in a model for Parkinson's disease. Cell Death Differ. 2012;19(3):416-27. doi:10. 1038/cdd.2011.105.

37. Song BL, Javitt NB, DeBose-Boyd RA. Insig-mediated degradation of HMG CoA reductase stimulated by lanosterol, an intermediate in the synthesis of cholesterol. Cell Metab. 2005; 1(3):179-89. doi:10.1016/j.cmet.2005.01.001.

38. Foresti O, Ruggiano A, Hannibal-Bach HK, Ejsing CS, Carvalho P. Sterol homeostasis requires regulated degradation of squalene monooxygenase by the ubiquitin ligase Doa10/Teb4. eLife. 2013;2:e0953.

39. Gonzalez R, Carlson JP, Dempsey ME. Two major regulatory steps in cholesterol synthesis by human renal cancer cells. Arch Biochem Biophys. 1979;196(2):574-80.

40. Hidaka Y, Satoh T, Kamei T. Regulation of squalene epoxidase in HepG2 cells. J Lipid Res. 1990;31(11):2087-94.

41. Gill S, Stevenson J, Kristiana I, Brown AJ. Cholesterol-dependent degradation of squalene monooxygenase, a control point in cholesterol synthesis beyond HMG-CoA reductase. Cell Metab. 2011;13(3):260-73. doi:10.1016/j.cmet.2011.01.015.

42. Zelcer N, Sharpe LJ, Loregger A, Kristiana I, Cook EC, Phan L, et al. The E3 ubiquitin ligase MARCH6 degrades squalene monooxygenase and affects 3-hydroxy-3-methyl-glutaryl coenzyme A reductase and the cholesterol synthesis pathway. Mol Cell Biol. 2014;34(7):1262-70. doi:10.1128/MCB.01140-13. 